\title{
Cross-validation of genes potentially associated with overall survival and drug resistance in ovarian cancer
}

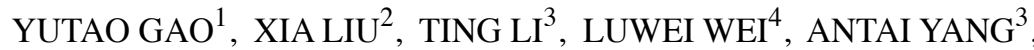 \\ YI LU ${ }^{2}$, JIAN ZHANG ${ }^{2}, \mathrm{LI} \mathrm{LI}^{4}$, SUMEI WANG $^{5}$ and FUQIANG $\mathrm{YIN}^{3,4}$ \\ ${ }^{1}$ Department of Gynaecology, First Affiliated Hospital of Kunming Medical University, Kunming, \\ Yunnan 650032; ${ }^{2}$ Center for Translational Medicine, Guangxi Medical University, Nanning, Guangxi 530021; \\ ${ }^{3}$ Life Sciences Institute, Guangxi Medical University, Nanning, Guangxi 530021; ${ }^{4}$ Key Laboratory of High-Incidence-Tumor \\ Prevention and Treatment (Guangxi Medical University), Ministry of Education, Nanning, Guangxi 530021; \\ ${ }^{5}$ The First Affiliated Hospital, Guangxi Medical University, Nanning, Guangxi 530021, P.R. China
}

Received October 2, 2016; Accepted March 15, 2017

DOI: 10.3892/or.2017.5534

\begin{abstract}
Ovarian cancer is the leading cause of death among malignancies of the female reproductive system. The 5-year survival rates of ovarian cancer (OC) patients are very poor as a result of recurrent disease and emergence of drug resistance; thus, studies to find predictive markers and factors for drug resistance are ongoing. In the present study, based on the microarrays from The Cancer Genome Atlas (TCGA), and the Gene Expression Omnibus (GEO) profiles covering 1648 OC patients, 11 out of 136 genes that were found to be significantly dysregulated in OC were associated with overall survival (OS) in 489 OC patients of the TCGA cohort. Of these genes, CRISP3, LYVE1, OVGP1 and BCHE were identified as independent prognostic factors, with decreased expression of the first three genes predicting shorter OS, and decreased $B C H E$ predicting longer OS. OVGP1, BCHE and further two genes, $C K A P 2$ and $C L D N 10$, were consistently and remarkably associated with OS when the number of patients increased from 489 to 1583 , with increased $C K A P 2$ and decreased $C L D N 10$ predicted shorter OS; combining the four genes provided better predictions. Associations among the four genes with OS in subgroups of OC were further verified. Downregulation of $O V G P 1$ was significantly associated with shorter OS in all subgroups of OC patients, including
\end{abstract}

Correspondence to: Professor Sumei Wang, The First Affiliated Hospital, Guangxi Medical University, 22 Shuangyong Road, Nanning, Guangxi 530021, P.R. China

E-mail: 187176076@qq.com

Professor Fuqiang Yin, Life Sciences Institute, Guangxi Medical University, 22 Shuangyong Road, Nanning, Guangxi 530021, P.R. China

E-mail: yinfq@mail2.sysu.edu.cn

Key words: ovarian cancer, overall survival, drug resistance, microarray subgroups of 752 patients treated with chemotherapy regimens containing taxol, 763 with both platin and taxol, 1364 with platin, 371 patients with grade 1-2 disease, 968 with grade 3 disease, 1148 with stage III-IV disease, and 439 with TP53 mutations. In addition, $C K A P 2$ expression was significantly associated with shorter OS in 515 OC patients who had low CA125 levels. Furthermore, comprehensive analyses that including RT-qPCR, bioinformatics analysis and clinical data revealed an association of CKAP2, BCHE, CLDN1O and $O V G P 1$ with drug resistance in $\mathrm{OC}$. The genes identified in the present study might be prognostic factors as well as potential therapeutic targets in the treatment of OC.

\section{Introduction}

Ovarian cancer is the most lethal cancer among gynecologic malignancies (1). Early-stage ovarian cancer is frequently asymptomatic and difficult to detect, and thus diagnosis usually occurs after the disease has disseminated beyond the ovaries (2). The current standard treatment for advanced ovarian cancer is surgical debulking followed by platinum/ taxane-based chemotherapy (3). Although this standard treatment significantly reduces the mortality rates and prolongs the survival time, the majority of patients will eventually relapse (4). The main obstacle to a successful treatment for ovarian cancer is the development of drug resistance that finally leads to fatal disease (5), and 5-year survival rates of ovarian cancer are less than $40 \%$, with only modest improvement over the past 40 years (2). Therefore, there is a consistent and urgent need to understand the mechanism of drug resistance and to identify useful biomarkers for overall survival in ovarian cancer (6).

The present study was based on profiles of 1648 ovarian cancer patients, in microarrays from The Cancer Genome Atlas (TCGA) and Gene Expression Omnibus (GEO). We identified several genes significantly associated with overall survival (OS); thus, these genes are potential biomarkers for prognosis in OC. We also found that these genes were potentially involved in regulation of drug resistance in OC. 


\section{Materials and methods}

Cell culture. The human epithelial OC cell lines SKOV3 and A2780 were maintained in our laboratory and propagated in vitro by serial passage in RPMI-1640 medium supplemented with $10 \%$ fetal bovine serum (FBS). The cisplatin-resistant cell line SKOV3-DDP and the carboplatin-resistant cell line A2780-CBP were established by sequential exposure of cells to increasing concentrations of cisplatin and carboplatin, respectively (7).

Real-time quantitative polymerase chain reaction analysis. Total RNA was isolated from the cell lines SKOV3, SKOV3-DDP, A2780 and A2780-CBP, using TRIzol reagent (Life Technologies, Grand Island, NY, USA). The quantity and quality of the RNA were measured using a Thermo Scientific NanoDrop 2000 spectrophotometer (Thermo Fisher Scientific, Wilmington, DE, USA). cDNA was synthesized from $2 \mu \mathrm{g}$ of RNA using the SuperScript III First-Strand synthesis system (Life Technologies). mRNA expression was measured using real-time quantitative polymerase chain reaction (RT-qPCR) and the Power SYBR-Green PCR Master Mix (Applied Biosystems, Waltham, MA, USA). Data were collected with the Applied Biosystems StepOne RT-PCR system in accordance with the manufacturer's instructions. The RT-qPCR gene-specific primers were: $C A K P 2$ : forward primer, 5'-AA GCCACAAAACCTCAGCCT-3' and reverse primer, 5'-CA TGAGGCCCTTTCCGGATT-3'. BCHE: forward primer, 5'-GGCCTGTCTTCAAAAGCACTG-3' and reverse primer, 5'-TCCTGCTTTCCACTCCCATTC-3'. CLDN10: forward primer, 5'-GGAGGCTCCGATAAAGCCAA-3' and reverse primer, 5'-GTGGCCCCGTTGTATGTGTA-3'. OVGP1: forward primer, 5'-AGCGAAGAAGCACTGGATTGA-3' and reverse primer, 5'-ATTCACAGCAGATGACAGCCA-3'. For $G A P D H$, used as the control, the forward primer was 5'-GA GGTGAAGGTCGGAGT-3' and the reverse primer 5'-GAA GATGGTGATGGGATTT-3'.

Gene expression profiles. Gene expression data and survival information of the 1648 OC patients were deposited in the KM plotter, which was established based on the microarrays from GEO and TCGA (8). Twelve independent microarrays from GEO profiles covered 1083 patients, including GSE14764 $(n=80)$, GSE15622 $(n=36)$, GSE18520 $(n=63)$, GSE19829 $(n=28)$, GSE23554 $(n=28)$, GSE26193 $(n=107)$, GSE26712 $(\mathrm{n}=195), \operatorname{GSE} 27651(\mathrm{n}=49)$, GSE30161 $(\mathrm{n}=58)$, GSE3149 $(n=166)$, GSE51373 $(n=28)$ and GSE9891 $(n=285)$; the TCGA ovarian cohort covered 565 patients. Detailed information of gene expression and clinical characteristics of the 565 OC patients in TCGA cohort were retrieved from the cBioPortal for cancer genomics (http://cbioportal.org) $(9,10)$.

Microarray GDS3754 (Human U133 plus 2.0 GeneChips; Affymetrix, Inc., Santa Clara, CA, USA) was deposited in GEO Profiles (11), and used for the analyses of gene expression in drug-resistant $\mathrm{OC}$ cells. In this analysis, in the case of two probe sets that target one gene, only the probe set with significant variability was retained. In the case of more than three probe sets that target one gene, the set exhibiting the most divergent expression was excluded and the set with significant variability was retained (12).
The TCGA ovarian cohort and GEO profiles are well known publicly available cohorts that can be downloaded by researchers for further analysis. No changes were made to mRNA expression values used in the analysis.

Bioinformatics analysis. Protein/gene interaction network was generated using the GeneMANIA (http://www.genemania. org/), which is a web-based database and a tool for the prediction of gene functions on the basis of multiple networks derived from different genomic or proteomic data/sources (13). It is fast enough to predict gene functions with great accuracy using GeneMANIA because hundreds of datasets from GEO, BioGRID, Pathway Commons and I2D, as well as organismspecific functional genomics data sets have been collected in this software (14).

Biological process annotation was performed using the Coremine Medical (http://www.coremine.com/medical/), which is a gene/protein database and a web-based tool for text mining. It carries out automated extraction of experimental and theoretical biomedical knowledge from publicly available gene and text databases to create a gene-to-gene co-citation network for millions of named human genes by automated analysis of titles and abstracts in over 10 million MEDLINE records (15).

Data analysis. The data were analyzed using SPSS 20.0 software. The probability of survival and significance were calculated using the Kaplan-Meier method and KM plotter (8). The correlation between gene expression and the clinicopathological characteristics was evaluated by Pearson's $\chi^{2}$ test (two-sided). Gene expression was dichotomized into high and low values using the median as a cut-off in all above analyses, in accordance with previous studies (16). Gene mRNA expression levels are shown as mean \pm SD. Homogeneity of the variance was analyzed using the t-test. $\mathrm{P}<0.05$ was considered significant. The desired Affymetrix ID for CKAP2, BCHE, CLDN10, and OVGP1 is 218252_at, 205433_at, 205328_at, and 205432_at, respectively.

\section{Results}

Determination of genes associated with $O S$ in $O C$. We previously reported that 60 genes were upregulated, and 126 genes were downregulated by at least 4-fold in 594 ovarian serous cystadenocarcinomas compared with 8 normal ovaries, respectively, according to TCGA ovarian statistics data deposited in the Oncomine database (17). Of these, we selected 136 genes (38 upregulated and 98 downregulated genes) for which we found $\leq 2$ articles in PubMed using a search with (ovarian[Title]) and 'gene'[Title/Abstract], for further analysis of their relationships with OS. The mRNA expression data of the 136 genes and related clinical data for 489 OC patients in the TCGA cohort were retrieved from cBioPortal for Cancer Genomics $(9,10)$. Gene expression was dichotomized into high and low values using the median as a cut-off in all above analyses, in accordance with a previous study (16). As shown in Table I, among all 136 genes, 11 were closely associated with OS, as determined by Kaplan-Meier analysis. $C K A P 2$ is upregulated, and ATXN10, BCHE, CLDN10, CRISP3, FCGBP, $L Y V E 1, N D N F, O V G P 1, P T G I S$ and REEPI are downregulated 
Table I. Eleven genes were determined by Kaplan-Meier analysis to be closely associated with overall survival in ovarian cancer.

\begin{tabular}{|c|c|c|c|c|c|c|}
\hline \multirow[b]{2}{*}{ Dysregulation of genes } & & \multirow[b]{2}{*}{ Estimate } & \multirow[b]{2}{*}{ Std. Error } & \multicolumn{2}{|c|}{$95 \% \mathrm{CI}$} & \multirow[b]{2}{*}{ P-value } \\
\hline & & & & Lower & Upper & \\
\hline \multicolumn{7}{|l|}{ Upregulated genes } \\
\hline \multirow[t]{3}{*}{$C K A P 2$} & $\mathrm{H}$ & 39.900 & 2.482 & 35.035 & 44.765 & 0.024 \\
\hline & $\mathrm{L}$ & 47.500 & 3.060 & 41.503 & 53.497 & \\
\hline & Overall & 43.800 & 2.105 & 39.675 & 47.925 & \\
\hline \multicolumn{7}{|l|}{ Downregulated genes } \\
\hline \multirow[t]{3}{*}{ ATXN10 } & $\mathrm{H}$ & 41.000 & 2.782 & 35.547 & 46.453 & 0.03 \\
\hline & $\mathrm{L}$ & 45.300 & 2.671 & 40.066 & 50.534 & \\
\hline & Overall & 43.800 & 2.105 & 39.675 & 47.925 & \\
\hline \multirow[t]{3}{*}{ BCHE } & $\mathrm{H}$ & 36.300 & 2.283 & 31.826 & 40.774 & 0.000 \\
\hline & $\mathrm{L}$ & 49.000 & 3.206 & 42.717 & 55.283 & \\
\hline & Overall & 43.800 & 2.105 & 39.675 & 47.925 & \\
\hline \multirow[t]{3}{*}{ CLDN10 } & $\mathrm{H}$ & 48.300 & 1.797 & 44.777 & 51.823 & 0.036 \\
\hline & $\mathrm{L}$ & 38.200 & 2.075 & 34.134 & 42.266 & \\
\hline & Overall & 43.800 & 2.105 & 39.675 & 47.925 & \\
\hline \multirow[t]{3}{*}{ CRISP3 } & $\mathrm{H}$ & 47.700 & 1.795 & 44.181 & 51.219 & 0.043 \\
\hline & $\mathrm{L}$ & 38.300 & 2.023 & 34.336 & 42.264 & \\
\hline & Overall & 43.800 & 2.105 & 39.675 & 47.925 & \\
\hline \multirow[t]{3}{*}{$F C G B P$} & $\mathrm{H}$ & 41.000 & 2.371 & 36.352 & 45.648 & 0.025 \\
\hline & $\mathrm{L}$ & 44.900 & 2.866 & 39.283 & 50.517 & \\
\hline & Overall & 43.800 & 2.105 & 39.675 & 47.925 & \\
\hline \multirow[t]{3}{*}{ LYVE1 } & $\mathrm{H}$ & 41.500 & 2.221 & 37.148 & 45.852 & 0.032 \\
\hline & $\mathrm{L}$ & 44.900 & 3.643 & 37.761 & 52.039 & \\
\hline & Overall & 43.800 & 2.105 & 39.675 & 47.925 & \\
\hline \multirow[t]{3}{*}{$N D N F$} & $\mathrm{H}$ & 41.000 & 2.070 & 36.944 & 45.056 & 0.034 \\
\hline & $\mathrm{L}$ & 45.100 & 2.086 & 41.012 & 49.188 & \\
\hline & Overall & 43.800 & 2.105 & 39.675 & 47.925 & \\
\hline \multirow[t]{3}{*}{$O V G P 1$} & $\mathrm{H}$ & 49.000 & 3.581 & 41.981 & 56.019 & 0.003 \\
\hline & $\mathrm{L}$ & 37.900 & 1.947 & 34.084 & 41.716 & \\
\hline & Overall & 43.800 & 2.105 & 39.675 & 47.925 & \\
\hline \multirow[t]{3}{*}{ PTGIS } & $\mathrm{H}$ & 40.400 & 2.890 & 34.735 & 46.065 & 0.004 \\
\hline & $\mathrm{L}$ & 47.500 & 1.975 & 43.629 & 51.371 & \\
\hline & Overall & 43.800 & 2.105 & 39.675 & 47.925 & \\
\hline \multirow[t]{3}{*}{ REEPI } & $\mathrm{H}$ & 38.400 & 2.053 & 34.377 & 42.423 & 0.007 \\
\hline & $\mathrm{L}$ & 47.700 & 3.021 & 41.779 & 53.621 & \\
\hline & Overall & 43.800 & 2.105 & 39.675 & 47.925 & \\
\hline
\end{tabular}

$\mathrm{H}$, high expression; L, low expression. Gene expression and survival data of 489 ovarian cancer patients in a TCGA cohort were used for the analysis. Expression values of a gene were dichotomized into high and low expression using the median as a cut-off.

in OC (17). We found that the upregulation of $C K A P 2$, and downregulation of CLDN10, CRISP3 and OVGP1 were associated with shorter OS, whereas the downregulation of ATXN10, BCHE, FCGBP, LYVE1, NDNF, PTGIS and REEPI were associated with longer OS. To elucidate whether any of the above genes was an independent factor for predicting OS, we performed multivariate analyses of histological grade, tumor stage, residual tumor and platinum status for the 11 genes, using a Cox proportional hazards model (Table II). The results indicated that BCHE $(\mathrm{P}=0.026)$, CRISP3 $(\mathrm{P}=0.031)$, LYVE1 $(\mathrm{P}=0.014)$ and $O V G P 1(\mathrm{P}=0.001)$ were independent prognostic factors for OS in 489 OC patients.

The associations of the 11 genes with OS were further validated using KM plotter which included gene expression data and survival information of 1287 OC patients downloaded from GEO and TCGA ovarian data in 2012 (8), and updated to 
Table II. Multivariate analysis of overall survival of ovarian cancer patients using Cox proportional hazard model.

\begin{tabular}{|c|c|c|c|c|c|c|c|c|}
\hline \multirow[b]{2}{*}{ Factors } & \multirow[b]{2}{*}{ B } & \multirow[b]{2}{*}{ SE } & \multirow[b]{2}{*}{ Wald } & \multirow[b]{2}{*}{ df } & \multirow[b]{2}{*}{ Sig. } & \multirow[b]{2}{*}{$\operatorname{Exp}(B)$} & \multicolumn{2}{|c|}{$95 \%$ CI } \\
\hline & & & & & & & Lower & Upper \\
\hline CKAP2 & 0.172 & 0.135 & 1.626 & 1 & 0.202 & 1.187 & 0.912 & 1.546 \\
\hline ATXN1O & 0.156 & 0.126 & 1.538 & 1 & 0.215 & 1.169 & 0.913 & 1.496 \\
\hline BCHE & 0.310 & 0.139 & 4.955 & 1 & 0.026 & 1.363 & 1.038 & 1.790 \\
\hline CLDN10 & -0.184 & 0.130 & 1.988 & 1 & 0.159 & 0.832 & 0.644 & 1.074 \\
\hline CRISP3 & -0.282 & 0.131 & 4.647 & 1 & 0.031 & 0.754 & 0.584 & 0.975 \\
\hline$F C G B P$ & 0.196 & 0.128 & 2.330 & 1 & 0.127 & 1.217 & 0.946 & 1.565 \\
\hline$L Y V E 1$ & 0.324 & 0.132 & 6.004 & 1 & 0.014 & 1.382 & 1.067 & 1.791 \\
\hline$N D N F$ & 0.057 & 0.132 & 0.184 & 1 & 0.668 & 1.058 & 0.818 & 1.370 \\
\hline$O V G P 1$ & -0.439 & 0.135 & 10.577 & 1 & 0.001 & 0.645 & 0.495 & 0.840 \\
\hline PTGIS & 0.030 & 0.142 & 0.045 & 1 & 0.832 & 1.030 & 0.781 & 1.360 \\
\hline REEPl & 0.075 & 0.132 & 0.325 & 1 & 0.568 & 1.078 & 0.833 & 1.395 \\
\hline $\begin{array}{l}\text { Grade } \\
\text { (G2/G3) }\end{array}$ & -0.279 & 0.197 & 2.010 & 1 & 0.156 & 0.756 & 0.514 & 1.113 \\
\hline $\begin{array}{l}\text { Platinum stat } \\
(\mathrm{R} / \mathrm{S})\end{array}$ & 1.230 & 0.168 & 53.690 & 1 & 0.000 & 3.420 & 2.462 & 4.753 \\
\hline $\begin{array}{c}\text { Residual (mr } \\
(\leq 10 />10)\end{array}$ & -0.176 & 0.145 & 1.482 & 1 & 0.223 & 0.838 & 0.631 & 1.113 \\
\hline $\begin{array}{l}\text { Stage } \\
\text { (II/III-IV) }\end{array}$ & -0.636 & 0.406 & 2.449 & 1 & 0.118 & 0.530 & 0.239 & 1.174 \\
\hline
\end{tabular}

$\mathrm{R} / \mathrm{S}$, platinum resistance/platinum sensitive. Gene expression and survival data of 489 ovarian cancer patients in a TCGA cohort were used for the analysis. Gene expression was dichotomized into high and low values using the median as a cut-off.
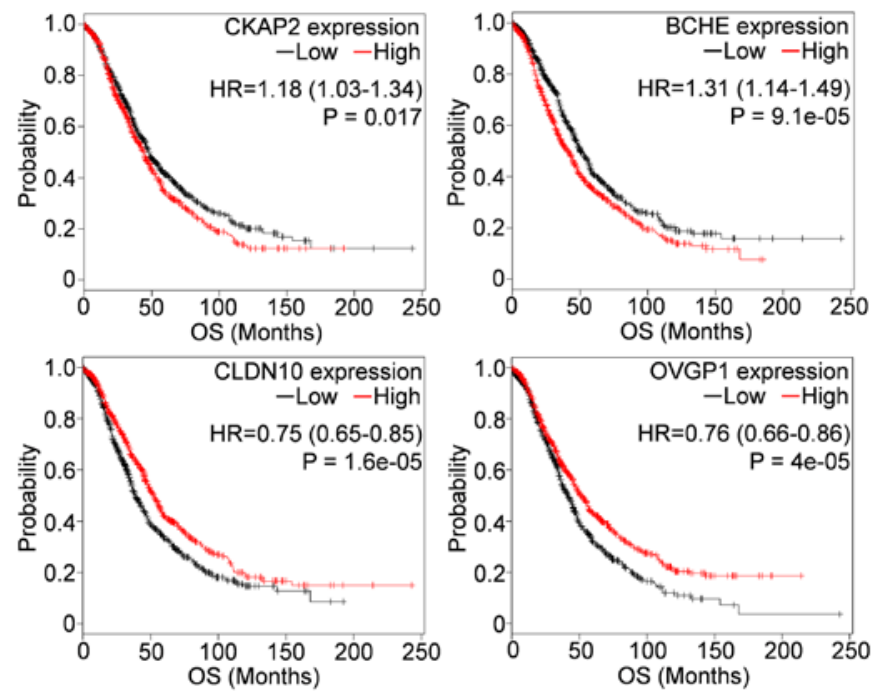

Figure 1. Relationships between gene expression and overall survival (OS) in 1583 OC patients, using KM plotter. Gene expression was dichotomized into high and low values using the median as a cut-off. HR, hazard ratio.

1648 patients for the 2015 version. As shown in Fig. 1, among the 11 genes, $C K A P 2, B C H E, C L D N 10$ and $O V G P 1$ were consistently associated with OS when the number of patients increased from 489 in TCGA cohort to 1583 in KM plotter (Table I and Fig. 1). Of these, upregulation of $C K A P 2$, and

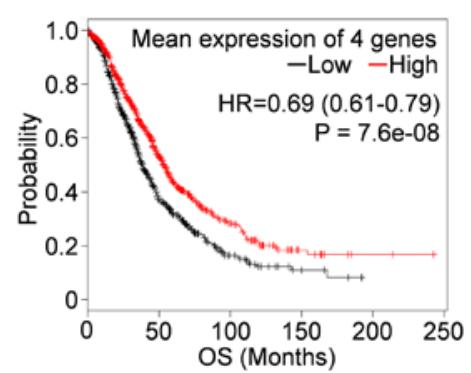

Figure 2. Relationships between mean expressions of the four genes and overall survival (OS) in 1583 OC patients, using KM plotter. Reciprocals of hazard ratio (HR) values (i.e., $H R^{-1}$ ) for $C K A P 2$ and $B C H E$ were used. Gene expression was dichotomized into high and low values using the median as a cut-off.

downregulation of $C L D N 10$ and $O V G P I$ were associated with shorter OS, whereas downregulation of $B C H E$ was associated with longer OS. When the four genes were combined, we found that their mean expression in combination was greatly more associated with OS than any of the genes used separately (Fig. 2).

Verification of genes associated with OS in OC patients treated with different chemotherapy regimens. As shown in Table III, in subgroups of patients treated with different regimens, we found that the high expression of $C K A P 2$ was associated with 
Table III. Four genes were associated with overall survival in subgroups of ovarian patients treated with different chemotherapy regiments, as determined by KM plotter.

Chemotherapy containing (no. of patients)

\begin{tabular}{|c|c|c|c|c|c|c|}
\hline \multirow[b]{2}{*}{ Gene } & \multicolumn{2}{|c|}{ Platin (1364) } & \multicolumn{2}{|c|}{ Taxol (780) } & \multicolumn{2}{|c|}{ Platin and taxol (763) } \\
\hline & $\mathrm{HR}(95 \% \mathrm{CI})$ & P-value & HR $(95 \% \mathrm{CI})$ & P-value & $\mathrm{HR}(95 \% \mathrm{CI})$ & P-value \\
\hline CKAP2 & $1.09(0.94-1.26)$ & 0.27 & $1.24(1.02-1.5)$ & 0.033 & $1.24(1.01-1.51)$ & 0.037 \\
\hline BCHE & $1.26(1.09-1.46)$ & 0.0017 & $1.19(0.97-1.44)$ & 0.088 & $1.19(0.97-1.45)$ & 0.091 \\
\hline CLDN10 & $0.76(0.66-0.88)$ & 0.00029 & $0.82(0.68-1)$ & 0.052 & $0.81(0.67-0.99)$ & 0.042 \\
\hline$O V G P 1$ & $0.77(0.66-0.89)$ & 0.00037 & $0.74(0.61-0.91)$ & 0.0031 & $0.74(0.61-0.91)$ & 0.0035 \\
\hline
\end{tabular}

Gene expression was dichotomized into high and low values using the median as a cut-off. HR, hazard ratio.
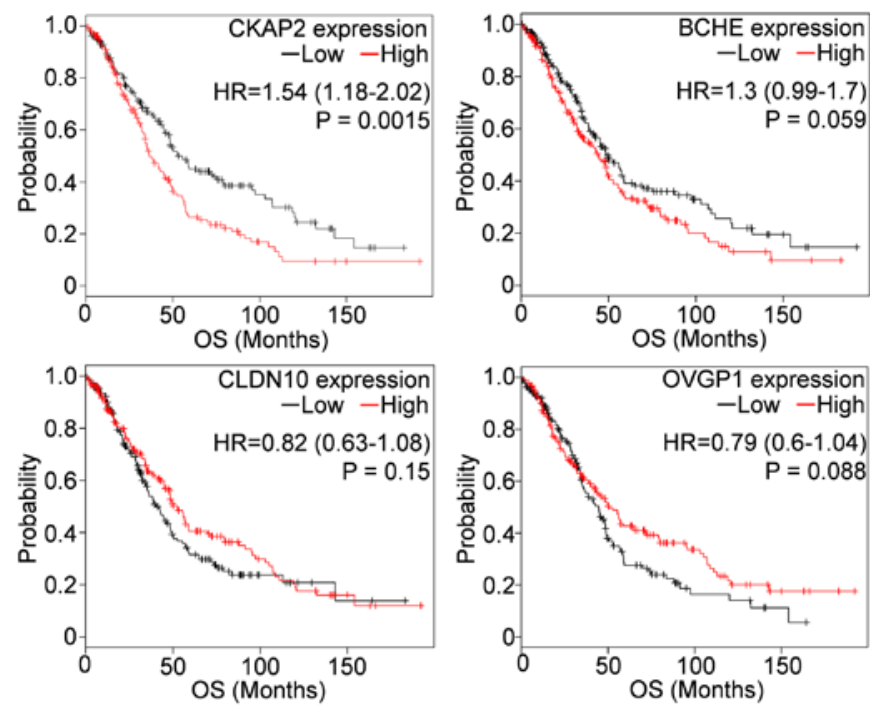

Figure 3. Relationships between gene expression and overall survival (OS) in 515 OC patients with average CA125 below lower quartile, using the KM plotter. Expression values of genes were dichotomized into high and low expressions using the median as a cut-off. HR, hazard ratio.

shorter OS in 752 patients treated with taxol-based regimens, and in 763 treated with regimens containing both platin and taxol, but the association was not significant in 1364 patient treated with only platins; downregulation of $B C H E$ predicts a longer OS in patients treated with platin, but not in patients treated with taxols or taxols with platin; downregulation of CLDN10 was associated with shorter OS in patients treated with platin or platin with taxol, but not in the taxol-only group, and downregulation of $O V G P 1$ predicted a shorter OS in all three subgroups of ovarian patients treated with different regimens.

Verification of genes associated with $O S$ in subgroups of $O C$ patients. The relationships between dysregulation of the four genes (CKAP2, BCHE, CLDN10 and OVGPI) with OS in subgroups of OC patients with different CA125 levels, grades, stages and TP53 mutation status were further investigated. We revealed that the high $C K A P 2$ expression significantly associated with shorter OS in 515 OC patients having low CA125
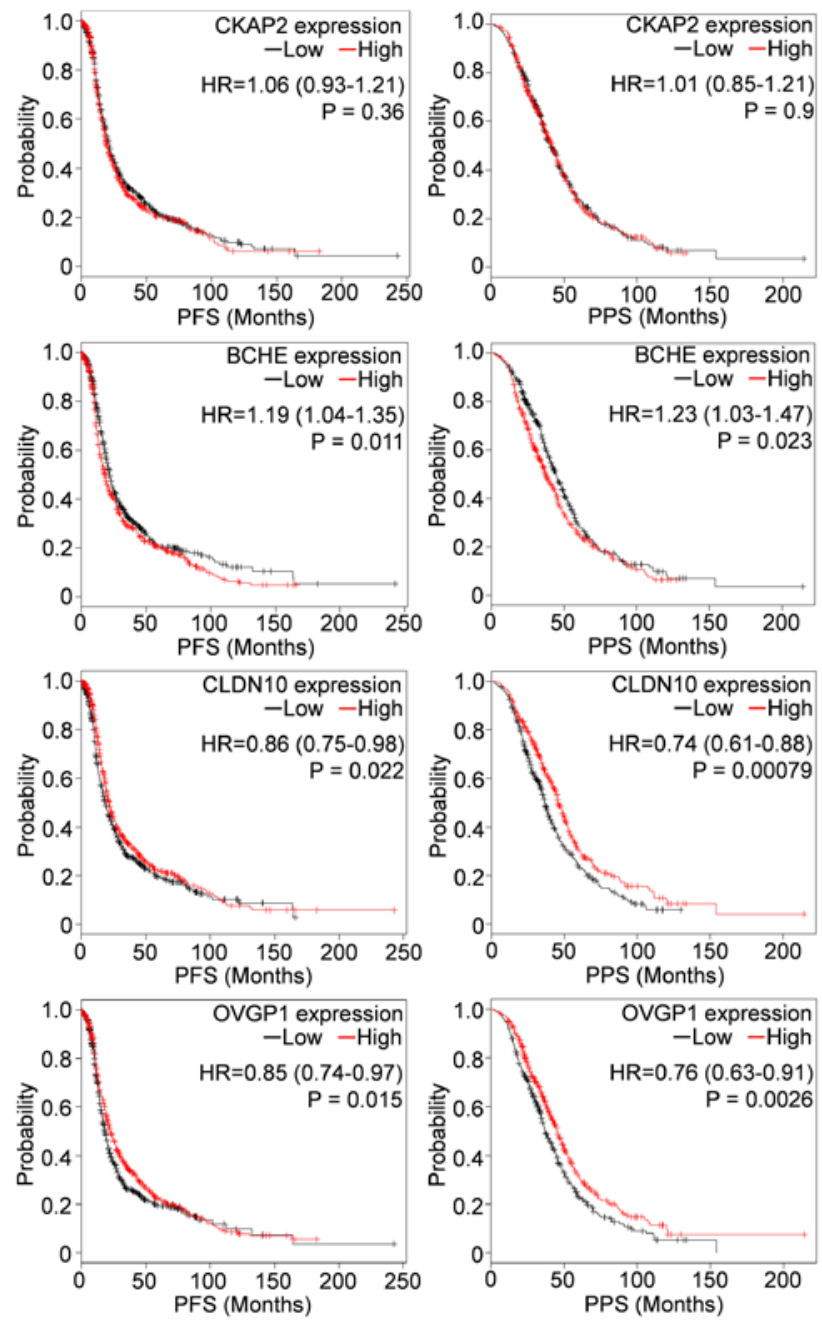

Figure 4. Relationships between gene expression in OC patients and progression-free survival (PFS; $n=1307$ ) or post-progression survival (PPS; $n=708$ ), as determined by KM plotter. Gene expression was dichotomized into high and low values using the median as a cut-off. HR, hazard ratio.

levels (the average CA125 below lower quartile) (Fig. 3). Besides, as shown in Table IV, all four genes were significantly associated with OS in 968 OC patients with grade 3 disease, but only $B C H E$ and $O V G P 1$ were associated with OS in 371 patients with grade 1-2 disease. CLDN10 and $O V G P 1$ were 
Table IV. The four genes were associated with overall survival in subgroups of ovarian patients with different grades, stages and TP53 mutation status, as determined by KM plotter.

\begin{tabular}{|c|c|c|c|c|c|c|c|c|c|}
\hline \multirow[b]{2}{*}{ Subgroup } & \multirow{2}{*}{$\begin{array}{c}\text { No. of } \\
\text { patients }\end{array}$} & \multicolumn{2}{|l|}{$C K A P 2$} & \multicolumn{2}{|l|}{ BCHE } & \multicolumn{2}{|l|}{ CLDN10 } & \multicolumn{2}{|l|}{$O V G P 1$} \\
\hline & & $\mathrm{HR}(95 \% \mathrm{CI})$ & P-value & $\mathrm{HR}(95 \% \mathrm{CI})$ & P-value & $\mathrm{HR}(95 \% \mathrm{CI})$ & P-value & $\mathrm{HR}(95 \% \mathrm{CI})$ & P-value \\
\hline \multicolumn{10}{|l|}{ Grade } \\
\hline I-II & 371 & $1.22(0.91-1.64)$ & 0.18 & $1.73(1.28-2.34)$ & 0.00026 & $0.78(0.58-1.04)$ & 0.091 & $0.58(0.43-0.78)$ & 0.00027 \\
\hline III & 968 & $1.21(1.02-1.43)$ & 0.032 & $1.20(1.01-1.42)$ & 0.04 & $0.75(0.63-0.89)$ & 0.00088 & $0.80(0.67-0.95)$ & 0.011 \\
\hline \multicolumn{10}{|l|}{ Stage } \\
\hline I-II & 133 & $1.36(0.61-3.01)$ & 0.45 & $1.04(0.47-2.31)$ & 0.92 & $0.90(0.41-1.97)$ & 0.78 & $0.70(0.32-1.55)$ & 0.38 \\
\hline III-IV & 1148 & $1.1(0.94-1.29)$ & 0.22 & $1.13(0.97-1.32)$ & 0.12 & $0.84(0.72-0.98)$ & 0.024 & $0.73(0.62-0.85)$ & $8.0 \mathrm{e}-5$ \\
\hline \multicolumn{10}{|l|}{ TP53 } \\
\hline Wild & 86 & $0.99(0.55-1.78)$ & 0.97 & $2.0(1.09-3.66)$ & 0.023 & $0.78(0.43-1.39)$ & 0.4 & $0.8(0.44-1.43)$ & 0.45 \\
\hline Mutation & 439 & $1.02(0.79-1.31)$ & 0.9 & $1.12(0.87-1.44)$ & 0.37 & $0.85(0.66-1.09)$ & 0.2 & $0.75(0.58-0.96)$ & 0.024 \\
\hline
\end{tabular}

Gene expression was dichotomized into high and low values using the median as a cut-off. HR, hazard ratio.

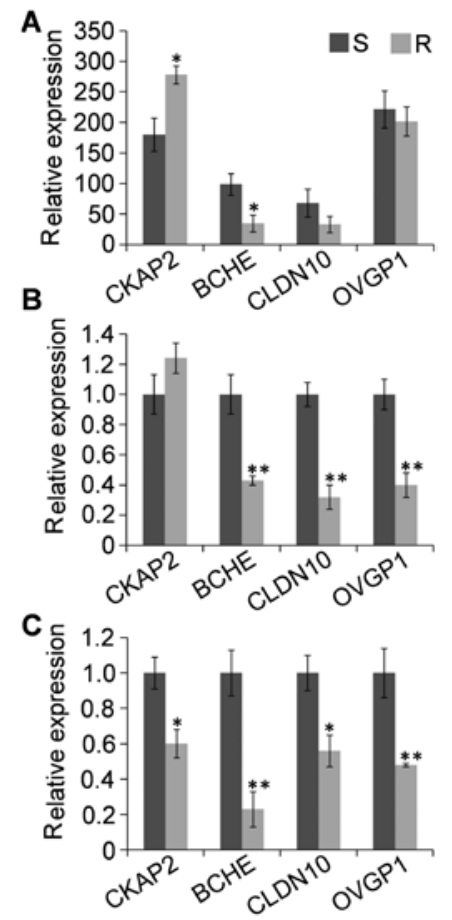

Figure 5. Gene mRNA expression in drug-sensitive and drug-resistant $\mathrm{OC}$ cells, using microarray data from GEO Profiles and RT-qPCR analysis. (A) mRNA expression of the four genes in drug-sensitive A2780 epithelial OC cells and cells with acquired platinum resistance, using microarray data (GDS3754). Five replicates were performed for each cell line. Normalized data were deposited in GEO Profiles (18). (B) mRNA expression of the four genes in cisplatin-sensitive and -resistant SKOV3 OC cells, as measured by RT-qPCR. Four replicates were performed for the drug-resistant cells and for the controls. (C) mRNA expression of the four genes in carboplatin-sensitive and -resistant A2780 OC cells, as measured by RT-qPCR. Four replicates were performed for the drug-resistant cells and for the controls. S, drug sensitive cells; R, drug resistant cells. ${ }^{*} \mathrm{P}<0.05 ;{ }^{* * *} \mathrm{P}<0.01$.

associated with OS in 1148 patients with stage III-IV OC, but none of the genes was associated with OS in 133 patients with stage I-II OC. In the subgroup of 439 patients with TP53 mutation, downregulation of $O V G P 1$ predicted shorter OS, whereas downregulation of $C L D N 10$ predicted shorter OS in the TP53 wild-type group. In addition, downregulation of $B C H E$ was associated with longer PFS and PPS in cohorts of 1307 and 708 patients, respectively; whereas downregulation of $C L D N 10$ and $O V G P 1$ were associated with shorter PFS and PPS (Fig. 4).

Clinical importance of the analysis. The four OS-related genes, $C K A P 2, B C H E, C L D N 10$ and $O V G P 1$, with clinical characteristics such as tumor stage, histological grade, and primary therapy outcome were checked in the TCGA cohort $(\mathrm{n}=489)$. As shown in Table $\mathrm{V}, B C H E$ and $O V G P 1$ were closely associated with primary therapy outcomes. Downregulation of $B C H E$ was associated with good complete response, whereas downregulation of $O V G P 1$ was associated with poor complete response. Besides, downregulation of $B C H E$ was associated with lower tumor stage, in particular, stage II. Only $C L D N 10$ was associated with platinum status; its downregulation might predict drug resistance. The 4 genes were not significantly associated with histological grade in this analysis.

Possible associations with drug resistance. The four genes, CKAP2, BCHE, CLDN10 and OVGP1, were dysregulated in platinum-resistant $\mathrm{OC}$ cells, as determined using microarray data retrieved from GEO Profiles and RT-qPCR analysis (Fig. 5). Compared with their expression in sensitive cells, expression of $C K A P 2$ was increased in cisplatin-resistant cells, but decreased in carboplatin-resistant cells; BCHE, CLDN1O and $O V G P 1$ all were decreased in cisplatin- and carboplatinresistant cells, although the changes in $C L D N 10$ and $O V G P I$ were not significant in one analysis (Fig. 5A).

Bioinformatics analyses were performed to further explain the associations of the four genes with drug resistance in OC. As shown in Fig. 6A, text mining indicated that BCHE had direct associations with drug resistance and $\mathrm{OC}$, and all the four genes were indirectly associated with drug resistance and OC through six biological processes $(\mathrm{P}<0.05)$, including cell 


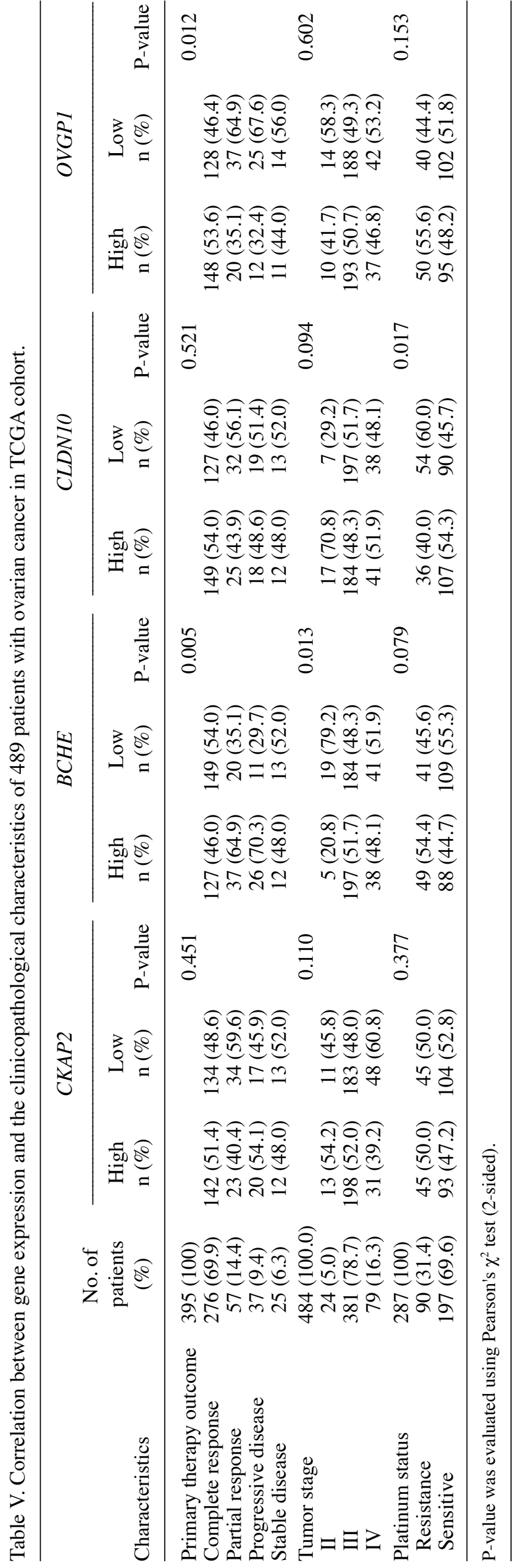

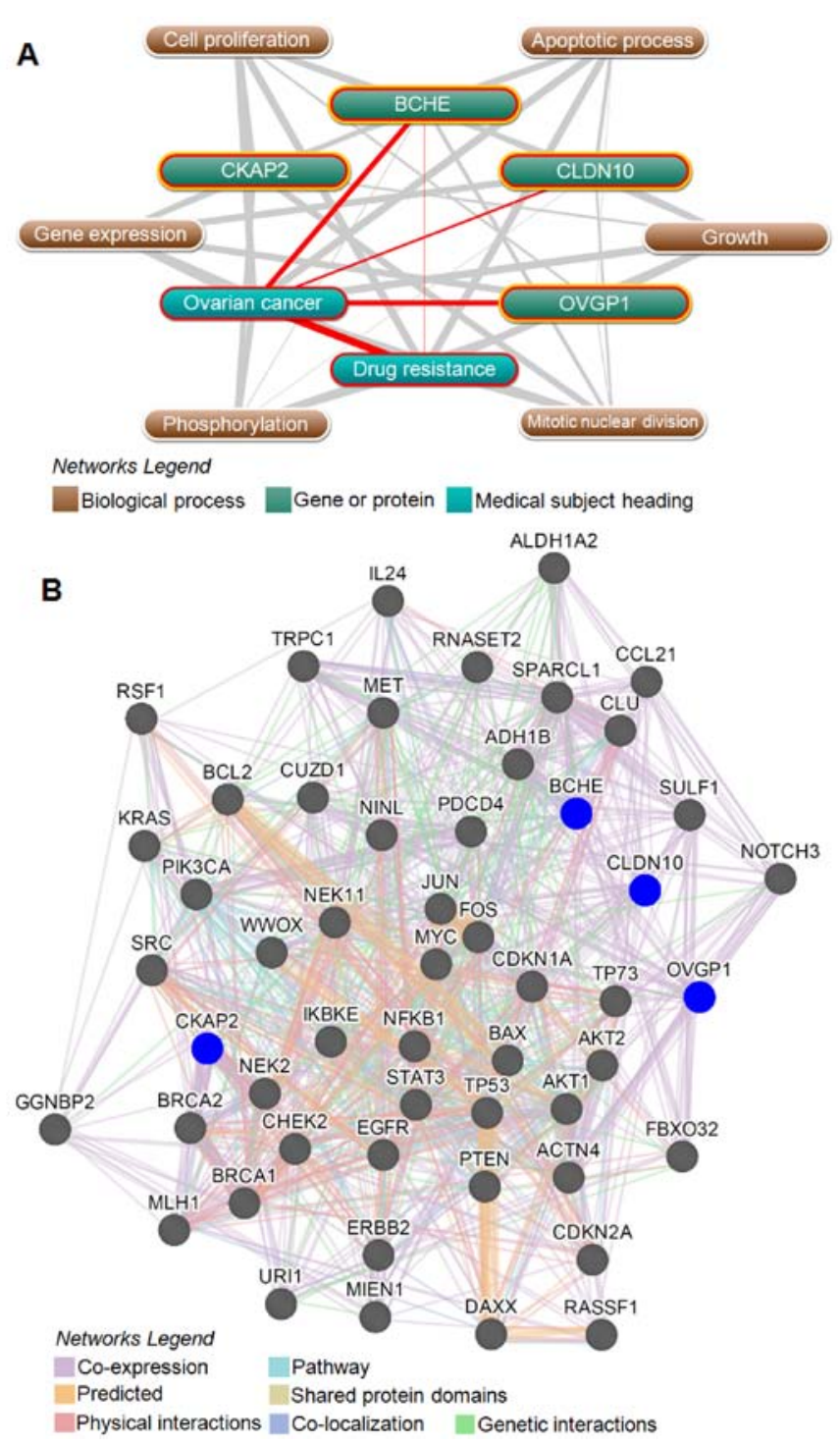

Figure 6. Bioinformatics analysis of the CKAP2, BCHE, CLDN10 and OVGP1 with drug resistance in OC. (A) Associations of the four genes/proteins with drug resistance and $\mathrm{OC}$, as determined using the Coremine Medical tool. Input terms were CKAP2, BCHE, CLDN10, OVGP1, drug resistance and OC. Red line, direct interactions of the input terms; grey line, direct interactions of the input terms with annotated biological processes $(\mathrm{P}<0.05)$; (B) protein/gene-protein/gene interaction network of the four proteins/genes with 49 drug resistance-related proteins/genes in OC (GeneMANIA tool). The query in blue includes the targets. The types of interactions between proteins/genes are indicated in the network legend of the figure.

proliferation, apoptosis, mitotic nuclear division, phosphorylation, growth and gene expression. We further generated a protein/gene interaction network for the four genes and their products with 49 drug-resistance proteins/genes in OC, which including 15 tumor suppressors (19): BRCA1, BRCA2, CHEK2, FBXO32, MLH1, SULF1, IL24, CDKN2A, CDKN1A, TP53, TP73, PDCD4, PTEN, RASSF1 and WWOX, 25 oncoproteins (20): ACTN4, AKT1, AKT2, BAX, BCL2, MIEN1, CLU, CUZD1, DAXX, EGFR, ERBB2, FOS, JUN, IKBKE, KRAS, MET, MYC, NFKB1, NINL, NOTCH3, PIK3CA, RSF1, SRC, STAT3 and URI1, NEK2 (21), NEK11 (22), TRPC1 (12), CCL21 and SPARCL1 (23), GGNBP2 and RNASET2 (7), ALDH1A2 and ADH1B (17). As shown in Fig. 6B, CKAP2, BCHE, CLDN10 and OVGP1 had direct and 
indirect interactions with all of the 49 drug-resistant proteins/ genes, and directly interacted with 11, 23, 19 and 13 of those 49 proteins/genes, respectively. The four genes and their products also directly interacted with each other, with $O V G P 1$ and $B C H E$ both directly interacting with $C K A P 2$ and $C L D N 10$.

\section{Discussion}

Biomarkers not only have prognostic implications, but are also helpful in monitoring treatment responses, surveillance for tumor recurrence and guidance of clinical decisions (24). Long-term survival of OC patients remains very poor as a result of recurrence and emergence of drug resistance, and the 5 -year survival rates is only $\sim 40 \%$ (2). Thus, prognostic biomarkers for OC patients are particularly necessary and crucial, and there is ongoing search for predictive biomarkers. In the present study, we identified a group of genes significantly associated with OS in 489 OC patients from a TCGA cohort (Table I), of which BCHE, CRISP3, LYVE1 and OVGPI were identified as independent risk prognostic factors for OS (Table II). Four genes (CKAP2, BCHE, CLDN10 and OVGPI) were consistently associated with OS when the number of patients increased from 489 to 1583 (Fig. 1). Of these, upregulation of $C K A P 2$, and downregulation of CLDN10 and $O V G P 1$ were associated with shorter OS, whereas downregulation of $B C H E$ was associated with longer OS. With the exception of $C K A P 2$, the other three genes all were significantly related to PFS and PPS, with decreased BCHE expression associated with longer PFS and PPS in 1307 and 708 OC patients, respectively; and decreased expression of CLDN10 and OVGP1 were associated with shorter PFS and PPS (Fig. 4). In subgroups of patients with $\mathrm{OC}$, we further verified that downregulation of $O V G P 1$ was significantly associated with shorter OS in all OC subgroups, including the 752 patients treated with chemotherapy regimens that contained taxol, 763 treated with both platin and taxol, 1364 treated with platin, 371 patients with grade 1-2 disease, 968 with grade 3 disease, 1148 with stage III-IV disease, and 439 with TP53 mutations (Tables III and IV). All these results together suggest that $C K A P 2, B C H E$, $C L D N 10$ and $O V G P 1$, (especially $O V G P 1$ ), are potential biomarkers for predicting $\mathrm{OS}$ in $\mathrm{OC}$.

The relationship between these genes and prognosis in $\mathrm{OC}$ is poorly studied. Upregulation of chromatin CKAP2 is an independent prognostic marker for shorter relapse-free survival in early-stage breast cancer (25), and for significantly worse prognoses in male patients with T1-2 gastric cancer (26). Downregulation of CLDN10 indicates shorter OS in some patients with lung adenocarcinoma (27). All these reports were consistent with our findings in this study. However, low levels of preoperative serum $B C H E$ were validated as an independent negative prognostic factor for prostate cancer patients who undergo radical prostatectomy (28), and a predictor of shorter OS and DFS in patients with muscle-invasive bladder cancer who undergo radical cystectomy (29). These results were contrary to our findings that downregulation of $\mathrm{BCHE}$ was associated with longer OS. The associations of the four genes with OS in OC is rare, with only one study reporting that the downregulation of $B C H E$ predicts a longer OS in $\mathrm{OC}$ patients, which was also based on the analysis of data in the TCGA cohort (30).
Serum CA125 is widely used to distinguish malignant from benign pelvic masses, to monitor response to treatment, to assess prognosis and to detect disease recurrence. Low levels of CA125 are normally an optimistic indicator of OC progression and prognosis. For example, preoperative CA125 $<65 \mathrm{U} / \mathrm{ml}$ correlates with longer survival in OC patients (31); CA125 <50 U/ml correlates with significantly longer DFS and OS in patient with borderline ovarian tumors (32). However, no data are available that further predicts OS in subgroups of patients with low levels of CA125. CKAP2 expression was significantly associated with shorter OS in 515 OC patients who had low CA125 levels (the average CA125 below lower quartile) (Fig. 3), which suggests that this gene could be used for prognosis prediction with CA125. However, this study measured tissue levels of CA125, whereas the FDA-approved test for OC is serum-based (8). Associations between CKAP2 and OS in patients with low CA125 levels should be further studied.

The relationships of the four genes with drug resistance in cancer has not been widely reported. In the present study, we found that $C K A P 2, B C H E, C L D N 10$ and $O V G P 1$ all were significantly dysregulated in cisplatin- and carboplatin-resistant cells (Fig. 5). Text mining indicated that the four genes were directly and indirectly associated with drug resistance through several biological processes. A protein/gene interaction network indicated that the four genes interacted with 49 drug-resistant proteins/genes in OC. In particular, $B C H E$ and CLDN10 directly interacted with 23 and 19 of those 49 proteins/genes (Fig. 6). Downregulation of CLDN10 was also an indicator of drug resistance, and downregulation of $O V G P 1$ was significantly associated with poor complete response (Table IV). All these results imply that dysregulation of the four genes affects drug resistance in OC. Furthermore, among patients treated with different regimens, high expression of $C K A P 2$ was associated with shorter OS in patients treated with taxol-containing regimens, whereas downregulation of $B C H E$ was associated with OS in patient treated by regimens without taxol; downregulation of CLDN10 was associated with shorter OS in patients treated with platin, and downregulation of $O V G P 1$ predicted a shorter OS in patients treated with either platin or taxol (Table III). These results indicated that $C K A P 2$ is associated with taxol resistance, $B C H E$ and $C L D N 10$ with platin resistance, and $O V G P 1$ with both platin and taxol resistance.

The roles of $C K A P 2, B C H E, C L D N 10$ and $O V G P 1$ in OC have rarely been studied. We previously reported that $C K A P 2$ was upregulated, and $B C H E, C L D N 10$ and $O V G P 1$ were downregulated in OC, by a 4.26, -8.39, -7.04 and -102.95-fold changes, respectively (17). Downregulation of $O V G P 1$ in $\mathrm{OC}$ was recently confirmed in a study that showed the protein to be less abundant in high-grade serous ovarian tumor fluids (malignant) than in benign serous cystadenoma tumor fluids (33). CLDN10 mRNA was specifically detected in five cancerous ovaries compared with three normal controls in experimental hens, which suggests that CLDN10 is a novel biomarker for detecting $\mathrm{OC}$ in chickens (34); however, its association with human OC was not discussed.

In summary, we identified 11 genes significantly related to OS in 489 OC patients. Of these, BCHE, CRISP3, LYVE1 and $O V G P 1$ were identified as independent risk prognostic 
factors. Four genes $C K A P 2, B C H E, C L D N 10$, and particularly the $O V G P 1$, were remarkably associated with OS in total of 1583 OC patients, and combination of these genes had much better prediction potential. Besides, comprehensive analysis indicated that $C K A P 2, B C H E, C L D N 10$ and $O V G P 1$ might contribute to drug resistance in OC. This study has implicated genes that might be both prognostic markers and potential therapeutic targets to pursue in the treatment of ovarian cancer.

\section{Acknowledgements}

This study was supported by the National Natural Science Foundation of China (grant nos. 81302283,81560424,81660606 and 81460397), the China Postdoctoral Science Foundation (nos. 2014M552535XB and 2014M552291), the Natural Science Foundation of Guangxi (nos. 2014GXNSFCA118010, 2015GXNSFBA139115，2015GXNSFAA139151 and 2014GXNSFBA118155).

\section{References}

1. Coleman RL, Monk BJ, Sood AK and Herzog TJ: Latest research and treatment of advanced-stage epithelial ovarian cancer. Nat Rev Clin Oncol 10: 211-224, 2013.

2. Siegel RL, Miller KD and Jemal A: Cancer statistics, 2016. CA Cancer J Clin 66: 7-30, 2016.

3. Vaughan S, Coward JI, Bast RC Jr, Berchuck A, Berek JS, Brenton JD, Coukos G, Crum CC, Drapkin R, Etemadmoghadam D, et al: Rethinking ovarian cancer: Recommendations for improving outcomes. Nat Rev Cancer 11: 719-725, 2011.

4. Pliarchopoulou K and Pectasides D: Epithelial ovarian cancer: Focus on targeted therapy. Crit Rev Oncol Hematol 79: 17-23, 2011.

5. Cannistra SA: Cancer of the ovary. N Engl J Med 351: 2519-2529, 2004.

6. Holohan C, Van Schaeybroeck S, Longley DB and Johnston PG: Cancer drug resistance: An evolving paradigm. Nat Rev Cancer 13: 714-726, 2013

7. Yin F, Liu L, Liu X, Li G, Zheng L, Li D, Wang Q, Zhang W and Li L: Downregulation of tumor suppressor gene ribonuclease $\mathrm{T} 2$ and gametogenetin binding protein 2 is associated with drug resistance in ovarian cancer. Oncol Rep 32: 362-372, 2014.

8. Gyorffy B, Lánczky A and Szállási Z: Implementing an online tool for genome-wide validation of survival-associated biomarkers in ovarian-cancer using microarray data from 1287 patients. Endocr Relat Cancer 19: 197-208, 2012.

9. Cerami E, Gao J, Dogrusoz U, Gross BE, Sumer SO, Aksoy BA, Jacobsen A, Byrne CJ, Heuer ML, Larsson E, et al: The cBio cancer genomics portal: An open platform for exploring multidimensional cancer genomics data. Cancer Discov 2: 401-404, 2012.

10. Gao J, Aksoy BA, Dogrusoz U, Dresdner G, Gross B, Sumer SO, Sun Y, Jacobsen A, Sinha R, Larsson E, et al: Integrative analysis of complex cancer genomics and clinical profiles using the cBioPortal. Sci Signal 6: pl1, 2013.

11. Edgar R, Domrachev $M$ and Lash AE: Gene Expression Omnibus: NCBI gene expression and hybridization array data repository. Nucleic Acids Res 30: 207-210, 2002.

12. Liu X, Zou J, Su J, Lu Y, Zhang J, Li L and Yin F: Downregulation of transient receptor potential cation channel, subfamily $\mathrm{C}$, member 1 contributes to drug resistance and high histological grade in ovarian cancer. Int J Oncol 48: 243-252, 2016.

13. Mostafavi S, Ray D, Warde-Farley D, Grouios C and Morris Q: GeneMANIA: A real-time multiple association network integration algorithm for predicting gene function. Genome Biol 9 (Suppl 1): S4, 2008.

14. Warde-Farley D, Donaldson SL, Comes O, Zuberi K, Badrawi R, Chao P, Franz M, Grouios C, Kazi F, Lopes CT, et al: The GeneMANIA prediction server: Biological network integration for gene prioritization and predicting gene function. Nucleic Acids Res 38 (Web Server): W214-W220, 2010.
15. Jenssen TK, Laegreid A, Komorowski J and Hovig E: A literature network of human genes for high-throughput analysis of gene expression. Nat Genet 28: 21-28, 2001.

16. Hedditch EL, Gao B, Russell AJ, Lu Y, Emmanuel C, Beesley J, Johnatty SE, Chen X, Harnett P, George J, et al; Australian Ovarian Cancer Study Group: ABCA transporter gene expression and poor outcome in epithelial ovarian cancer. J Natl Cancer Inst 106: 106, 2014.

17. Liu X, Gao Y, Zhao B, Li X, Lu Y, Zhang J, Li D, Li L and Yin F: Discovery of microarray-identified genes associated with ovarian cancer progression. Int J Oncol 46: 2467-2478, 2015.

18. Li M, Balch C, Montgomery JS, Jeong M, Chung JH, Yan P, Huang TH, Kim S and Nephew KP: Integrated analysis of DNA methylation and gene expression reveals specific signaling pathways associated with platinum resistance in ovarian cancer. BMC Med Genomics 2: 34, 2009.

19. Yin F, Liu X, Li D, Wang Q, Zhang W and Li L: Tumor suppressor genes associated with drug resistance in ovarian cancer (Review). Oncol Rep 30: 3-10, 2013.

20. Liu X, Gao Y, Lu Y, Zhang J, Li L and Yin F: Oncogenes associated with drug resistance in ovarian cancer. J Cancer Res Clin Oncol 141: 381-395, 2015.

21. Liu X, Gao Y, Lu Y, Zhang J, Li L and Yin F: Upregulation of NEK2 is associated with drug resistance in ovarian cancer. Oncol Rep 31: 745-754, 2014.

22. Liu X, Gao Y, Lu Y, Zhang J, Li L and Yin F: Downregulation of NEK11 is associated with drug resistance in ovarian cancer. Int $J$ Oncol 45: 1266-1274, 2014.

23. Yin F, Liu X, Li D, Wang Q, Zhang W and Li L: Bioinformatic analysis of chemokine (C-C motif) ligand 21 and SPARC-like protein 1 revealing their associations with drug resistance in ovarian cancer. Int J Oncol 42: 1305-1316, 2013.

24. Wong KF, Xu Z, Chen J, Lee NP and Luk JM: Circulating markers for prognosis of hepatocellular carcinoma. Expert Opin Med Diagn 7: 319-329, 2013 .

25. Kim HS, Koh JS, Choi YB, Ro J, Kim HK, Kim MK, Nam BH, Kim KT, Chandra V, Seol HS, et al: Chromatin CKAP2, a new proliferation marker, as independent prognostic indicator in breast cancer. PLoS One 9: e98160, 2014.

26. Kim YW, Eom BW, Kook MC, Kim HS, Kim MK, Hwang HL, Chandra V, Poojan S, Song Y, Koh JS, et al: Clinical implications of proliferation activity in T1 or T2 male gastric cancer patients. Exp Mol Med 47: e193, 2015.

27. Zhang Z, Wang A, Sun B, Zhan Z, Chen $\mathrm{K}$ and Wang C: Expression of CLDN1 and CLDN10 in lung adenocarcinoma in situ and invasive lepidic predominant adenocarcinoma. J Cardiothorac Surg 8: 95, 2013.

28. Koie T, Ohyama C, Hatakeyama S, Imai A, Yoneyama T, Hashimoto Y, Yoneyama T, Tobisawa Y, Hosogoe S, Yamamoto H, et al: Significance of preoperative butyrylcholinesterase as an independent predictor of biochemical recurrence-free survival in patients with prostate cancer treated with radical prostatectomy. Int J Clin Oncol 21: 379-383, 2016.

29. Koie T, Ohyama C, Yamamoto H, Hatakeyama S, Imai A, Yoneyama $\mathrm{T}$, Hashimoto $\mathrm{Y}$, Kitayam $\mathrm{M}$ and Hirota $\mathrm{K}$ : Significance of preoperative butyrylcholinesterase as an independent predictor of survival in patients with muscle-invasive bladder cancer treated with radical cystectomy. Urol Oncol 32: 820-825, 2014.

30. Willis S, Villalobos VM, Gevaert O, Abramovitz M, Williams C, Sikic BI and Leyland-Jones B: Single gene prognostic biomarkers in ovarian cancer: A meta-analysis. PLoS One 11: e0149183, 2016.

31. Nagele F, Petru E, Medl M, Kainz C, Graf AH and Sevelda P: Preoperative CA 125: An independent prognostic factor in patients with stage I epithelial ovarian cancer. Obstet Gynecol 86: $259-264,1995$

32. Tang A, Kondalsamy-Chennakesavan S, Ngan H, Zusterzeel P, Quinn M, Carter J, Leung Y and Obermair A: Prognostic value of elevated preoperative serum CA125 in ovarian tumors of low malignant potential: A multinational collaborative study (ANZGOG0801). Gynecol Oncol 126: 36-40, 2012.

33. Poersch A, Grassi ML, Carvalho VP, Lanfredi GP, Palma CS, Greene LJ, de Sousa CB, Carrara HH, Candido Dos Reis FJ and Faça VM: A proteomic signature of ovarian cancer tumor fluid identified by highthroughput and verified by targeted proteomics. J Proteomics 145: 226-236, 2016.

34. Seo HW, Rengaraj D, Choi JW, Ahn SE, Song YS, Song G and Han JY: Claudin 10 is a glandular epithelial marker in the chicken model as human epithelial ovarian cancer. Int J Gynecol Cancer 20: 1465-1473, 2010. 